\title{
Certified Clinical Research Professional
}

National Cancer Institute

\section{Source}

National Cancer Institute. Certified Clinical Research Professional. NCI Thesaurus. Code C71302.

A clinical research professional (i.e., administrator, coordinator, consultant, educator, or researcher in clinical trial management) with related work experience and with certification earned by passing a required program and written examination from the Society of Clinical Research Associates (SOCRA) or equivalent organization. 